\title{
Robust Rydberg gate via Landau-Zener control of Förster resonance
}

\author{
Xi-Rong Huang, ${ }^{1}$ Zong-Xing Ding, ${ }^{1}$ Chang-Sheng Hu, ${ }^{1}$ Li-Tuo Shen, ${ }^{1}$ Weibin $\mathrm{Li},{ }^{2,3}$ Huaizhi Wu, ${ }^{1,2,3,{ }^{*}}$ and Shi-Biao Zheng ${ }^{1, \dagger}$ \\ ${ }^{1}$ Fujian Key Laboratory of Quantum Information and Quantum Optics and Department of Physics, Fuzhou University, \\ Fuzhou, Fujian 350116, China \\ ${ }^{2}$ School of Physics and Astronomy, University of Nottingham, Nottingham NG7 2RD, United Kingdom \\ ${ }^{3}$ Centre for the Mathematics and Theoretical Physics of Quantum Non-equilibrium Systems, University of Nottingham, \\ Nottingham NG7 2RD, United Kingdom
}

(Received 11 July 2018; published 19 November 2018)

\begin{abstract}
In this paper, we propose a scheme to implement the two-qubit controlled- $Z$ gate via the Stark-tuned Förster interaction of Rydberg atoms, where the Förster defect is driven by a time-dependent electric field of a simple sinusoidal function while the matrix elements of the dipole-dipole interaction are time independent. It is shown that when the system is initially in a specific state, it makes a cyclic evolution after a preset interaction time, returning to the initial state, but picks up a phase, which can be used for realizing a two-atom controlled- $Z$ gate. Due to the interference of sequential Landau-Zener transitions, the population and phase of the state is quasideterministic after the cyclic evolution and therefore the gate fidelity is insensitive to fluctuations of the interaction time and the dipole-dipole matrix elements. Feasibility of the scheme realized with Cs atoms is discussed in detail, which shows that the two-qubit gate via Landau-Zener control can be realized with the state-of-the-art experimental setup.
\end{abstract}

DOI: 10.1103/PhysRevA.98.052324

\section{INTRODUCTION}

Rydberg atoms trapped in optical potentials provide an attractive physical architecture for quantum information processing [1]. Long-range interactions between distant Rydberg atoms can be switched on and off on demand with focused lasers [2]. After the pioneering work proposed by Jaksch et al. [3], a number of schemes have been proposed to implement quantum gates with Rydberg atoms using, e.g., full and partial blockade, as well as antiblockade [4-20]. A useful way for controlling the interaction is Stark-tuned Förster resonance [21], where two pairs of Rydberg states that allow dipole transitions in between can be shifted into resonance by dc or microwave electric fields [22,23]. The coherent coupling at Förster resonance has been recently demonstrated in experiment $[24,25]$ and were earlier proposed for implementing quantum logic gates $[26,27]$. To achieve high-fidelity gates, one typically has to control Förster resonances precisely, which means these schemes are sensitive to fluctuations of interatomic distances and intensity of external fields.

To reduce the effect of parameter fluctuations in dynamical control, Beterov et al. have recently proposed a scheme for realizing a controlled- $Z(\mathrm{CZ})$ gate based on a double adiabatic passage across the Stark-tuned Förster resonance, enabling complete population transfer and accumulation of a deterministic phase for the targeted Rydberg pair state [28]. However, to avoid manipulating the distance-dependent matrix elements of the dipole-dipole interaction, the modulation function of the electric field applied for the Stark-tuning requires having

\footnotetext{
*huaizhi.wu@fzu.edu.cn

†sbzheng11@163.com
}

a power-law relation with respect to the gate operation time, which may increase experimental complexity nevertheless $[28,29]$.

Coherent population transfer in a two-level system can be realized alternatively via periodic sweeping of the interactioninduced avoided level crossing under the control of an external field, giving rise to the Landau-Zener (LZ) transitions [29-33] and the Landau-Zener-Stückelberg (LZS) oscillations [33]. The latter is also referred to as LZS interference since repeated passages through an avoided crossing act as an atomic interferometer [34], causing interference among different components of the atomic superposition state. If more than one crossing is involved and the dynamics is overall coherent, then transition paths can interfere according to the phases accumulated between subsequent crossings [35]. The LZ and LZS dynamics have been experimentally demonstrated with Rydberg atoms $[30-33,36]$.

Inspired by Ref. [28], we propose in this paper a scheme for implementation of two-qubit logic gates based on the LZ control of the Förster interaction. The dipole-dipole matrix elements for the coupling between the two Rydberg atoms remain constant during the gate operation, while the Förster defect is periodically modulated such that the interatomic interaction oscillates between van der Waals and dipolar shapes. The dynamics of the Rydberg pair states subjected to the Förster interaction is described by the LZS theory, and is discussed in strong, weak, and intermediate driving regimes. Our results show that a two-qubit CZ gate with high fidelity can be implemented based on a quasideterministic population transfer and phase accumulation, which can be much less sensitive to the fluctuations of the gate operation time and the dipole-dipole matrix elements compared with the scheme based on direct coherent coupling. Furthermore, 


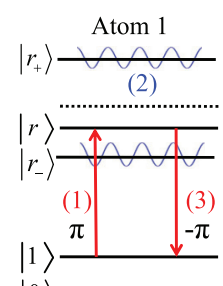

$|0\rangle$

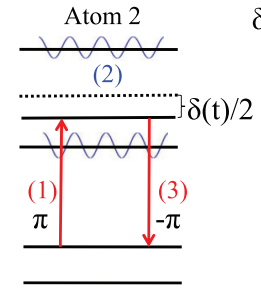

(a)

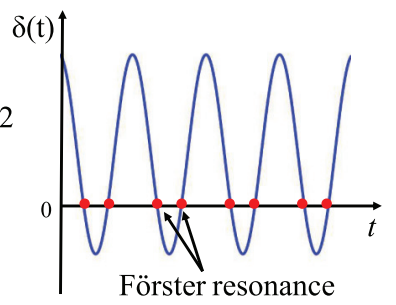

(b)
FIG. 1. (a) Scheme of a CZ gate based on Landau-Zener dynamics. Two atoms are first excited to the Rydberg state $|r\rangle$, followed by a harmonic driving that shifts the neighboring Rydberg levels $\left|r_{ \pm}\right\rangle$back and forth modulating the Förster resonance. The atoms are finally deexcited to the ground state $|1\rangle$. The phase shift is accumulated if both atoms are initially prepared in the state $|1\rangle$, and $|0\rangle$ is an auxiliary computational state. (b) Time dependence of the energy defect from the Förster resonance $\delta(t)=\delta_{0}+\hbar A \cos (\omega t+\phi)$, where the red dots denote the system passing through the Förster resonance induced by periodic modulation.

the adiabatic passage based scheme (cf. Ref. [28]) is implemented by sequentially applying two nonlinear driving pulses, whose intensities and durations need to be exactly identical and follow power-law dependence on time. Thus, it may be sensitive to time deviations, see further discussion in Sec. IV. However, the intensity of the driving field in the LZS-based scheme is a simple harmonic function of time and therefore the experimental complexity can be greatly reduced.

\section{MODEL AND SCHEME}

As shown in Fig. 1(a), we consider two identical Rydberg atoms individually trapped in optical tweezers. Each one has two ground states $|0\rangle$ and $|1\rangle$, which represent the logic states of the corresponding qubit, and three Rydberg states $|r\rangle,\left|r_{+}\right\rangle$, and $\left|r_{-}\right\rangle$. The transitions between the Rydberg levels $|r\rangle$ and $\left|r_{+}\right\rangle\left(\left|r_{-}\right\rangle\right)$are dipole allowed and the bare energies of the Rydberg pair states $|r\rangle_{1}|r\rangle_{2},\left|r_{+}\right\rangle_{1}\left|r_{-}\right\rangle_{2}$, and $\left|r_{-}\right\rangle_{1}\left|r_{+}\right\rangle_{2}$ are almost degenerate. The pair states are coupled by the dipolar interaction based on the Förster process

$$
|r\rangle_{1}|r\rangle_{2} \leftrightarrow\left|r_{+}\right\rangle_{1}\left|r_{-}\right\rangle_{2}+\left|r_{-}\right\rangle_{1}\left|r_{+}\right\rangle_{2},
$$

with the Rabi frequency (strength) $V_{D D} / 2$ and the Förster defect $\delta=E_{r_{+} r_{-}}-E_{r r}$, which can be modulated by an external electric field. Note that the two atoms excited to different Rydberg states (e.g., $\left|r_{a}\right\rangle$ and $\left|r_{b}\right\rangle$ ) may experience Förster resonances as well and the interaction channel will be revised as $\left|r_{a}\right\rangle_{1}\left|r_{b}\right\rangle_{2} \leftrightarrow\left|r_{+}\right\rangle_{1}\left|r_{-}\right\rangle_{2}$ (or $\left|r_{b}\right\rangle_{1}\left|r_{a}\right\rangle_{2} \leftrightarrow\left|r_{-}\right\rangle_{1}\left|r_{+}\right\rangle_{2}$ ) [23,28]. The Förster interactions can be found in both rubidium and cesium Rydberg atoms, see Sec. IV for further discussion.

The two-qubit controlled- $Z$ gate is implemented through LZ control of the Förster defect in three steps. Step (1): The two atoms are simultaneously excited to the Rydberg state $|r\rangle$ by a short $\pi$ pulse when they are in the state $|1\rangle$, and the electric field is tuned far away from Förster resonance [see Fig. 1(b)] so that the atomic pair transitions $|r\rangle_{1}|r\rangle_{2} \rightarrow$ $\left|r_{+}\right\rangle_{1}\left|r_{-}\right\rangle_{2}\left(\left|r_{-}\right\rangle_{1}\left|r_{+}\right\rangle_{2}\right)$ are adiabatically inhibited. Step (2): By applying a time-dependent sinusoidal electric field of radio frequency, the energy defect $\delta(t)$ is tuned to zero periodically and the system transits in between the pair of Rydberg states by passing through the avoided level crossing induced by the resonant dipole-dipole interaction (i.e., the Förster resonance). This results in coherent population transfer of the system states from $|r\rangle_{1}|r\rangle_{2}$ to $\left(\left|r_{+}\right\rangle_{1}\left|r_{-}\right\rangle_{2}+\left|r_{-}\right\rangle_{1}\left|r_{+}\right\rangle_{2}\right) / \sqrt{2}$ and then back to $|r\rangle_{1}|r\rangle_{2}$, accompanied by accumulation of a phase shift $\pi$. Note that the coherent population transfer can be realized as well for the energy defect $\delta(t)$ being much larger than the inherent dipole-dipole matrix elements; however, this is nonideal for realization of the $\mathrm{CZ}$ gate, see further discussion below. Step (3): A deexcitation pulse (the second $\pi$ pulse) is applied to the two atoms, transforming the doubly excitation state back to $|1\rangle_{1}|1\rangle_{2}$. Provided that one of the atoms is initially in the state $|0\rangle$, no phase shift can occur because Förster resonances are not present. Consequently, the system evolution is equivalent to the $\mathrm{CZ}$ gate:

$$
\begin{aligned}
& |0\rangle_{1}|0\rangle_{2} \longrightarrow|0\rangle_{1}|0\rangle_{2}, \quad|0\rangle_{1}|1\rangle_{2} \longrightarrow|0\rangle_{1}|1\rangle_{2}, \\
& |1\rangle_{1}|0\rangle_{2} \longrightarrow|1\rangle_{1}|0\rangle_{2}, \quad|1\rangle_{1}|1\rangle_{2} \longrightarrow-|1\rangle_{1}|1\rangle_{2} .
\end{aligned}
$$

\section{LZS CONTROL OF RYDBERG PAIR STATES}

To illustrate more clearly the realization of the essential transformation $|1\rangle_{1}|1\rangle_{2} \longrightarrow-|1\rangle_{1}|1\rangle_{2}$ (i.e., $|r\rangle_{1}|r\rangle_{2} \longrightarrow$ $-|r\rangle_{1}|r\rangle_{2}$ ), we reduce the Förster process by the coupling between two symmetric pair states for the two atoms: $|g\rangle \equiv$ $|r\rangle_{1}|r\rangle_{2}$ and $|e\rangle \equiv\left(\left|r_{+}\right\rangle_{1}\left|r_{-}\right\rangle_{2}+\left|r_{-}\right\rangle_{1}\left|r_{+}\right\rangle_{2}\right) / \sqrt{2}$. The Hamiltonian for the Förster defect being driven by a time-varying radio-frequency $(\mathrm{rf})$ field is then given by $(\hbar=1)$

$$
\hat{H}(t)=-\frac{1}{2}\left(\begin{array}{cc}
0 & V_{\mathrm{DD}} \\
V_{\mathrm{DD}} & 2 \delta(t)
\end{array}\right),
$$

with

$$
\delta(t)=\delta_{0}+A \cos (\omega t+\phi),
$$

where the bare energy of the state $|g\rangle$ is set to zero, and the coupling strength between the two newly defined basis states (i.e., the energy splitting of the avoiding crossing) is assumed to be independent of time. A key element here is the timevarying detuning $\delta(t)$, which is a periodic function with offset $\delta_{0}$, amplitude $A$, and frequency $\omega$. For simplicity, we take the phase $\phi=0$ in the following. The eigenenergies of $\hat{H}(t)$ under periodic modulation, which correspond to the frequencies of the two collective states $|+\rangle=\cos \theta(t)|e\rangle+\sin \theta(t)|g\rangle$ and $|-\rangle=\cos \theta(t)|g\rangle-\sin \theta(t)|e\rangle$ with $\theta(t)=\tan ^{-1}\left(V_{\mathrm{DD}} / \delta\right) / 2$, show avoided crossings while the Förster defect is tuned toward resonance, as shown in Fig. 2. The effective model (3) without involving pulse shaping of the coupled Rabi frequency is closely related to Rydberg experiments, where the matrix elements of the dipole-dipole coupling between two Rydberg atoms are determined by the interatomic distance and the orientation of the individual dipole, and cannot be continuously changed in short time scales.

We then rewrite the system Hamiltonian (3) by separating it into time-independent and time-dependent driving parts:

$$
\hat{H}(t)=\hat{H}_{0}+\hat{H}_{d}(t),
$$

with

$$
\begin{gathered}
\hat{H}_{0}=-\delta_{0}|e\rangle\langle e|-\frac{1}{2} V_{\mathrm{DD}}(|g\rangle\langle e|+| e\rangle\langle g|), \\
\hat{H}_{d}(t)=-A \cos \omega t|e\rangle\langle e| .
\end{gathered}
$$


In the rotating frame of $\hat{H}_{d}(t), \hat{H}_{0}$ can be transformed into

$$
\hat{H}^{\prime}(t)=\hat{U}(t) \hat{H}_{0} \hat{U}^{\dagger}(t)-i \hat{U}(t) \dot{\hat{U}}^{\dagger}(t)=-\frac{1}{2}\left(\begin{array}{cc}
0 & V_{\mathrm{DD}} e^{-i(A / \omega) \sin \omega t} \\
V_{\mathrm{DD}} e^{i(A / \omega) \sin \omega t} & 2 \delta_{0}
\end{array}\right),
$$

with the operator $\hat{U}(t)$ being

$$
\hat{U}(t)=\exp \left(-i \int_{0}^{t} \hat{H}_{d}(t) d t\right)=\exp \left[i\left(\frac{A}{\omega} \sin \omega t\right)|e\rangle\langle e|\right],
$$

which also maps the wave function in the reference frame $|\psi\rangle$ onto $\left|\psi^{\prime}\right\rangle$ via $\left|\psi^{\prime}\right\rangle=\hat{U}(t)|\psi\rangle$, following the Schrödinger equation $i \frac{d}{d t}\left|\psi^{\prime}\right\rangle=\hat{H}^{\prime}(t)\left|\psi^{\prime}\right\rangle$. The Hamiltonian (7) after making use of the Jacobi-Anger expansion

$$
e^{i x \sin \tau}=\sum_{n=-\infty}^{\infty} J_{n}(x) e^{i n \tau}
$$

takes the form

$$
\hat{H}^{\prime}(t)=-\frac{1}{2}\left(\begin{array}{cc}
0 & \sum_{n=-\infty}^{\infty} \Omega_{n} e^{-i n \omega t} \\
\sum_{n=-\infty}^{\infty} \Omega_{n}^{*} e^{i n \omega t} &
\end{array}\right)
$$

where the periodic energy defect has effectively modified the Rabi coupling $\Omega_{n}=V_{\mathrm{DD}} J_{n}\left(\frac{A}{\omega}\right)$ with $J_{n}\left(\frac{A}{\omega}\right)$ being the $n$ th-order Bessel function of the first kind. In addition, the resonance condition $\delta_{0}=m \omega$, which describes $|m|$-rf-photon transition process between the two collective states assisted by the rf driving field, can be identified by examining the time-independent term of the nondiagonal elements if the rotating wave approximation is made [37].

The LZ dynamics determined by $\hat{H}^{\prime}$ strongly depends on the driving parameters $A, \delta_{0}$, and $\omega$, which must satisfy two conditions to implement a robust $\mathrm{CZ}$ gate. First, the system should be able to make a full cycle of Rabi-like oscillation between the two collective states and accumulate a $\pi$ phase, which requires the Förster defect $\sim \delta_{0}$ to be an integer multiple of the rf-photon frequency for $\delta_{0} \gg V_{\mathrm{DD}}$, and the frequency of the oscillation $\sim V_{\mathrm{DD}} J_{n}(A / \omega)$ should be as large as possible such that the gate operation is decoherence resistant [38]. Second, the oscillatory period needs to be an integer multiple of the time period $\tau_{d}=2 \pi / \omega$ of a complete LZ passage, which makes the population and phase of the state $|g\rangle$ after the cyclic evolution robust to imperfect timing for a slow pas-

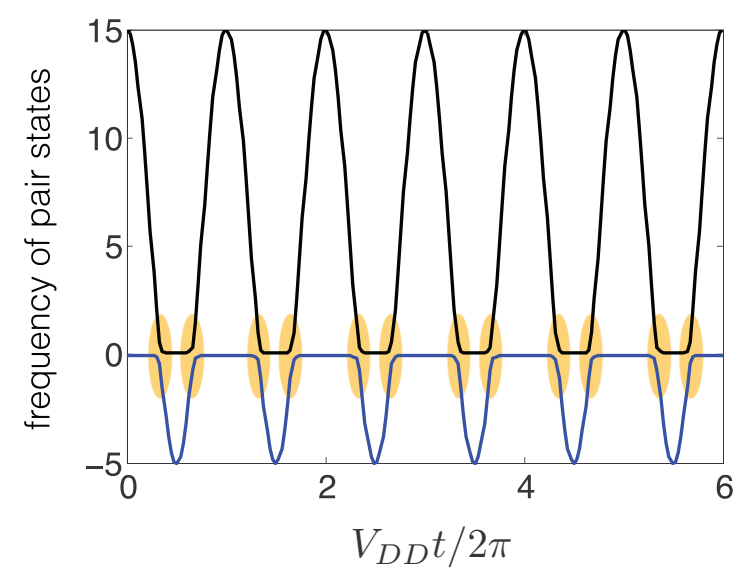

FIG. 2. Frequencies of the two collective states $| \pm\rangle$ as a function of rescaled time. The shaded regions indicate the avoided crossings resulting from resonant dipole-dipole (DD) interactions. Parameters are $V_{\mathrm{DD}}=1$ and $\left(A, \delta_{0}, \omega\right) / V_{\mathrm{DD}}=(10,5,1)$. sage $\left(\omega / V_{\mathrm{DD}} \sim 1\right)$. In the following, we study three different regimes of the driving parameters and focus on the situations that the Förster defect between the pair of Rydberg states is large compared with the dipole-dipole matrix elements (i.e. $\left.\delta_{0} \gg V_{\mathrm{DD}}\right)$.

Weak driving. The weak-driving regime refers to $A \ll$ $E_{q} \equiv \sqrt{\delta_{0}^{2}+V_{\mathrm{DD}}^{2}}$, under which, the single-rf-photon resonant transition from $|g\rangle$ to $|e\rangle$ occurs for $\omega=\sqrt{\delta_{0}^{2}+V_{\mathrm{DD}}^{2}}$ and the frequency of the Rabi oscillations is given by $\Omega_{e g} \equiv$ $A \sin \left[\tan ^{-1}\left(V_{\mathrm{DD}} / \delta_{0}\right)\right] / 2$. If the system is initially in the state $|g\rangle$ with a large static defect $\delta_{0} \gg V_{\mathrm{DD}}$, the valid approximation $\sin \left[\tan ^{-1}\left(V_{\mathrm{DD}} / \delta_{0}\right)\right] \approx V_{\mathrm{DD}} / \delta_{0}$ can be made to the Rabi frequency, giving rise to $\Omega_{e g} \approx A V_{\mathrm{DD}} / 2 \delta_{0}$. As a special case, this also describes the Autler-Townes splitting at small driving amplitude $A$, and can be analytically calculated by simply truncating the series of $\Omega_{n}$ up to $n=1$ (corresponding to the rotating wave approximation), which leads to [39]

$$
\begin{aligned}
\hat{H}^{\prime}(t) \approx & -\frac{1}{2}\left(\begin{array}{cc}
0 & V_{\mathrm{DD}} J_{0}\left(\frac{A}{\omega}\right) \\
V_{\mathrm{DD}} J_{0}\left(\frac{A}{\omega}\right) & 2 \delta_{0}
\end{array}\right) \\
& -\frac{1}{2}\left(\begin{array}{cc}
0 & V_{\mathrm{DD}} J_{1}\left(\frac{A}{\omega}\right) e^{i \omega t} \\
V_{\mathrm{DD}} J_{1}\left(\frac{A}{\omega}\right) e^{-i \omega t} & 0
\end{array}\right) .
\end{aligned}
$$

Using the interaction picture representation, we can then find the effective transition frequency $\sqrt{\delta_{0}^{2}+V_{\mathrm{DD}}^{2} J_{0}^{2}\left(\frac{A}{\omega}\right)}$ and the Rabi frequency $V_{\mathrm{DD}} J_{1}\left(\frac{A}{\omega}\right) \approx A V_{\mathrm{DD}} / 2 \delta_{0}$ of the two-level system by using the approximation $J_{n}\left(\frac{A}{\omega}\right) \sim \frac{(A / \omega)^{n}}{2^{n} n !}$ for $A / \omega \ll 1$. According to Eq. (10), if the system is initially in the state $|g\rangle$, it will undergo Rabi oscillations between $|g\rangle$ and $|e\rangle$ and return to $|g\rangle$ after a full Rabi cycle, but pick up a phase $\varphi$. This is illustrated in Fig. 3, but which shows $\varphi$ is generally not equal to $\pi$, so that a two-qubit controlled- $Z$ gate cannot be realized in this regime.

Strong driving. We next turn to the case of strong driving with $\left(A-\delta_{0}\right) \gg V_{\mathrm{DD}}$, where the system repeatedly traverses the Förster resonance and hardly spends any time in the degeneracy point [37]. To gain insight, we perform a further transformation $\exp \left(i \delta_{0}|e\rangle\langle e| t\right)$, transforming the Hamiltonian 

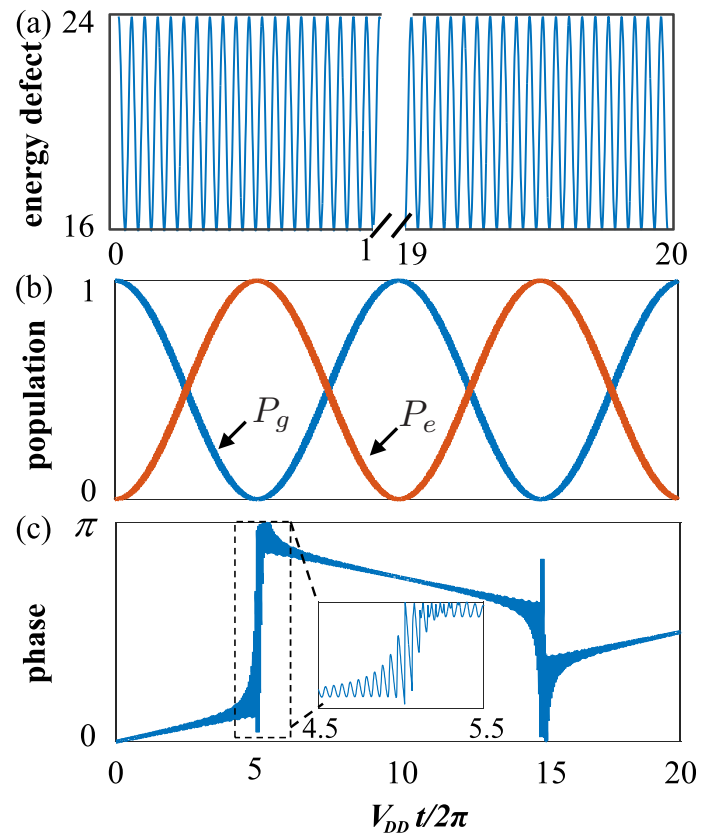

FIG. 3. (a) Time-dependent energy defect $\delta(t)$ as a function of dimensionless rescaled time $V_{\mathrm{DD}} t$. (b) Evolutions of the populations of the state $|g\rangle\left(P_{g}\right)$ and the excited state $|e\rangle\left(P_{e}\right)$ for the system initially in the state $|g\rangle$. (c) Time-dependent phase of the state $|g\rangle$. We fix units of $V_{\mathrm{DD}}=1$ and set $\left(A, \delta_{0}, \omega\right) / V_{\mathrm{DD}}=(4,20,20)$.

of Eq. (9) to

$$
\hat{H}_{I}(t)=-\frac{1}{2} \sum_{n=-\infty}^{\infty}\left(\begin{array}{cc}
0 & \Omega_{n} e^{i\left(\delta_{0}-n \omega\right) t} \\
\Omega_{n}^{*} e^{-i\left(\delta_{0}-n \omega\right) t} & 0
\end{array}\right) .
$$

Under the condition $\delta_{0}=m \omega$, the driving associated with the effective frequency component with $n=m$ is in resonance, corresponding to a $|m|$-rf-photon process. In the highfrequency limit, where the frequency of the external driving is much larger than the effective Rabi frequencies associated with the other frequency components, i.e., $\omega \gg \Omega_{n}(n \neq m)$, all time-dependent fast oscillating terms $\left(\sim e^{i(m-n) \omega t}\right)$ can be neglected. As a consequence, the system dynamics is reduced to the resonant driving of a two-level system with the Rabi frequency $\Omega_{n}=V_{\mathrm{DD}}\left|J_{n}\left(\frac{A}{\omega}\right)\right|$, as shown in Figs. 4(a)-4(c). In this case, the two-level system can make a cyclic evolution, and return to the initial state $|g\rangle$, picking up a phase of $\pi$. But this occurs almost at a specific moment $T_{c z} \sim 1 / V_{\mathrm{DD}}\left|J_{1}\left(\frac{A}{\omega}\right)\right|$ since the time interval between subsequent transition events is of the order of half the driving period $1 / 2 \omega$, which is short here. Thus, the evolutional dynamics analogous to the coherent resonant coupling scheme requires precise control of the rescaled time. In addition, we note that there is a special situation with the parameters $\delta_{0}=0$ and $n=0$, in which the system can transit between $|e\rangle$ and $|g\rangle$ with full conversion via the LZ control; however, here we focus on the general case of a finite Förster defect.

In the low-frequency situation $\omega \sim \Omega_{m}$, a stepwise increase or decrease of the population can be found for each time the system passing through the LZ avoid crossing and the population has weak oscillations during its stay at each stair. In general, the system exhibits nonsinusoidal oscillations and can approximately return to the initial state $|g\rangle$ with the quasideterministic population and phase $\pi$ after a time period $T$, which are exactly multiples of the driving period, e.g., $V_{\mathrm{DD}} T \sim 2 \pi \times 4$ with $T / \tau_{d}=12$ in Figs. 4(d) -4 (f) and $V_{\mathrm{DD}} T \sim 2 \pi \times 7$ with $T / \tau_{d}=5$ in Figs. 4(g)-4(i). The duration for the system staying in $|g\rangle$ after an oscillation period is again determined by the time interval for two sequential sweeping of the avoided crossing, as indicated in Figs. 4(g)4(i). Mathematically, this is because the single resonant transition with $\delta_{0}=m \omega$ is not a good approximation any more in the low-frequency regime, where the "noise channels" contribute to the Rabi coupling between the two basis states if the corresponding Rabi frequencies of the nonresonant
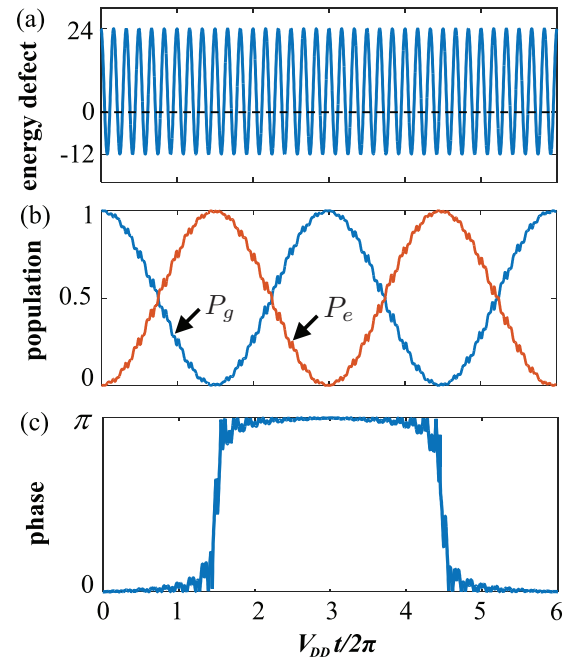

(d)

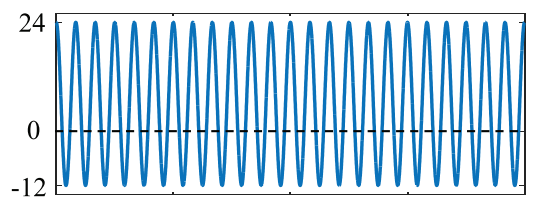

(e)

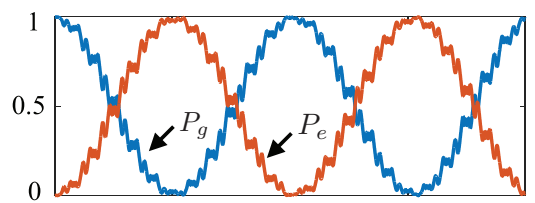

(f)

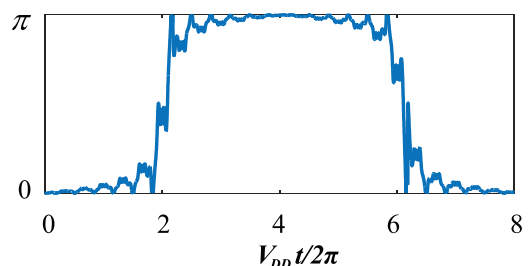

(g)

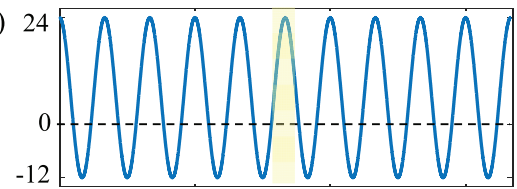

(h)

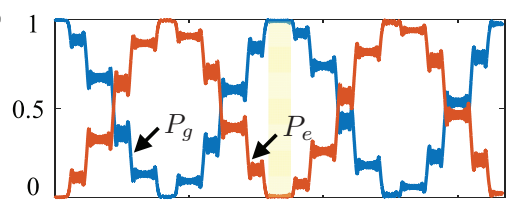

(i)

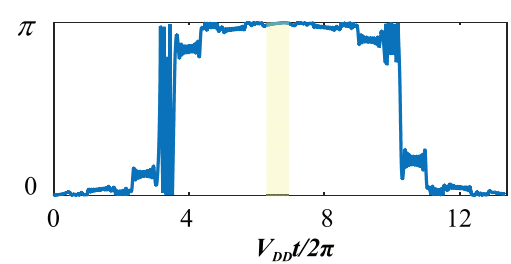

FIG. 4. (a), (d), (g) Time-dependent energy defect $\delta(t)$ as a function of dimensionless rescaled time $V_{\mathrm{DD}} t$. (b), (e), (h) Evolutions of the populations of the state $|g\rangle\left(P_{g}\right)$ and the excited state $|e\rangle\left(P_{e}\right)$ for the system initially in the state $|g\rangle$. (c), (f), (i) Time-dependent phase of the state $|g\rangle$. We fix units of $V_{\mathrm{DD}}=1$, and $\operatorname{set}\left(A, \delta_{0}, \omega\right) / V_{\mathrm{DD}}=(18,6,6)$ in (a)-(c), $\left(A, \delta_{0}, \omega\right) / V_{\mathrm{DD}}=(18,6,3)$ in (d)-(f), and $\left(A, \delta_{0}, \omega\right) / V_{\mathrm{DD}}=$ $(18,6,0.75)$ in $(\mathrm{g})-(\mathrm{i})$, respectively. 

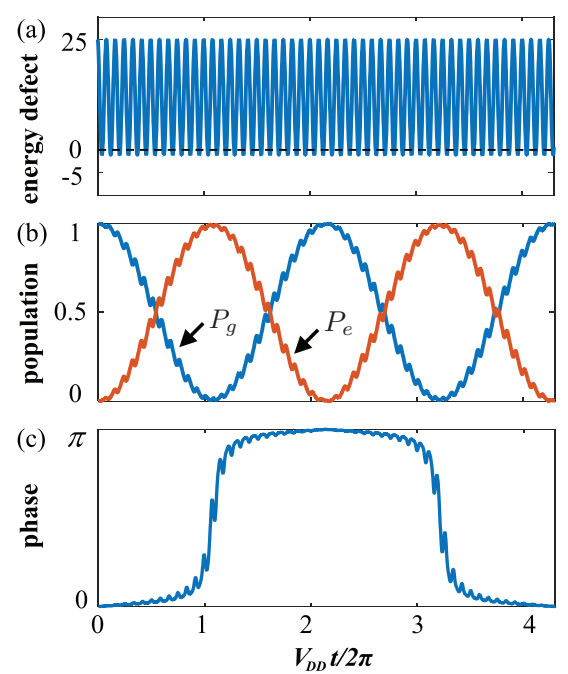

(d)

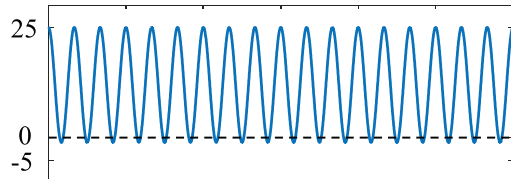

(e)

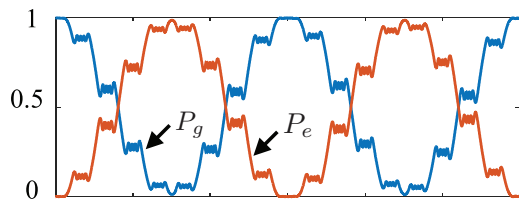

(f)

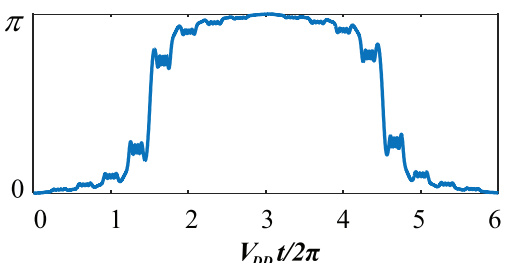

(g)

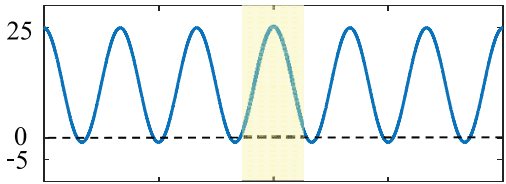

(h)

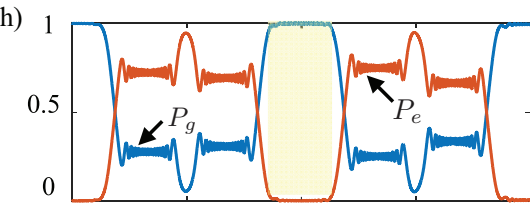

(i)

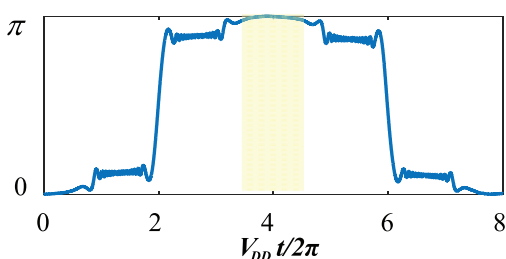

FIG. 5. (a), (d), (g) Time-dependent energy defect $\delta(t)$ as a function of dimensionless rescaled time $V_{\mathrm{DD}} t$. (b), (e), (h) Evolutions of the populations of the state $|g\rangle\left(P_{g}\right)$ and the excited state $|e\rangle\left(P_{e}\right)$ for the system initially in the state $|g\rangle$. (c), (f), (i) Time-dependent phase of the state $|g\rangle$. We fix units of $V_{\mathrm{DD}}=1$, and set $\left(A, \delta_{0}, \omega\right) / V_{\mathrm{DD}}=(13,12,12)$ in $(\mathrm{a})-(\mathrm{c}),\left(A, \delta_{0}, \omega\right) / V_{\mathrm{DD}}=(13,12,3)$ in $(\mathrm{d})-(\mathrm{f})$, and $\left(A, \delta_{0}, \omega\right) / V_{\mathrm{DD}}=$ $(13,12,0.75)$ in $(\mathrm{g})-(\mathrm{i})$, respectively.

components are comparable to the detunings, i.e., $(n-$ $m) \omega \sim \Omega_{n}$. This feature makes the gate dynamics robust against certain amounts of time deviation and parameter fluctuation.

Intermediate driving. Finally, we look into the regime with $A \simeq \delta_{0}$, where the system reaches the Förster resonance around the turning point of the harmonic driving, but stays for a longer time at the avoided crossing compared with the case of strong driving. To illustrate the performance of the controlled- $Z$ gates in this regime, we perform numerical simulations with three sets of parameters, as shown in Fig. 5. The results show the system exhibits the LZ transition behavior similar to that under strong driving for both high-frequency and slow-passage limits. The distinct features of the dynamics of the system in this regime are that it can return to the initial state $|g\rangle$ and pick up a $\pi$ phase after a relatively short time, and that the evolution can be frozen for a relatively long duration [see the shaded area in Figs. 5(g)-5(i)]. These features enable implementation of a high-fidelity controlled- $Z$ gate that is robust to parameter fluctuations.

The reason why the performance of the $\mathrm{CZ}$ gate in the intermediate-driving regime is better than that under strong driving can be explained as follows. On one hand, the avoided crossing is passed at a slower speed and the population exchange for each LZ passage is greatly enhanced. Therefore, the time period for the cyclic evolution and the corresponding gate operation time is shortened. The effective Rabi frequency for the cyclic evolution can be estimated by $\Omega_{\text {eff }} \sim$ $V_{\mathrm{DD}} J_{m}(A / \omega)$, which under the condition of $m=\delta_{0} / \omega \gg 1$ has a maximum around $A / \delta_{0} \sim 1$ [37]. On the other hand, the dynamics is robust to imperfect timing only in the lowfrequency driving limit $\omega / V_{\mathrm{DD}} \sim 1$ and is, however, bounded by the requirement of an integer number of $T / \tau_{d} \sim \omega / \Omega_{\text {eff }}$. Thus, in the intermediate-driving regime [see Figs. 5(g)-5(i)], the time evolution of the population and phase can be frozen for almost a complete driving period $2 \pi / \omega$ under the minimal driving frequency $\omega / \Omega_{\text {eff }} \sim 3$. As a consequence, the system does not oscillate back and forth between the two collective states for a smaller driving frequency.

To compare the stabilities of the gates realized in the strong- and intermediate-driving regimes, we plot the corresponding populations of the state $|g\rangle$ and the acquired phases against the time deviation in Fig. 6. The results show that both the population fluctuation and phase fluctuation in the intermediate-driving regime are smaller than those in the strong-driving regime. In the case of intermediate driving with $\delta\left(V_{\mathrm{DD}} T\right) /\left(V_{\mathrm{DD}} T\right) \sim 10 \%$, we find that the population fluctuation is less than $2 \%$. On the other hand, the phase acquired in the strong-driving regime oscillates between $0.98 \pi$ and $\pi$ within this time deviation, but the phase error remains less than $0.02 \pi$.

\section{IMPLEMENTATION OF THE CONTROLLED-Z GATE AND EXPERIMENTAL FEASIBILITY}

Now we focus on the intermediate-driving regime, which allows the optimal control of the population transfer and phase accumulation. To evaluate the performance of the controlled- $Z$ gate (2), we take an example where the input state is $\left|\psi_{0}\right\rangle=$ $\frac{1}{2}(|00\rangle+|01\rangle+|10\rangle+|11\rangle)$. The quality of the output state $\left|\psi_{f}\right\rangle$ under nonideal conditions is characterized by the fidelity, defined as $F=\left|\left\langle\psi_{f}\left|U_{\mathrm{CZ}}\right| \psi_{0}\right\rangle\right|^{2}$ where $U_{\mathrm{CZ}}$ is a diagonal matrix with $\operatorname{diag}\left(U_{\mathrm{CZ}}\right)=\left(1,1,1, e^{i \pi}\right)$. We first consider the effect of parameter fluctuation and neglect the atomic spontaneous emission. Assume that both the first and third steps of the controlled- $Z$ gate operation (i.e., the excitation and deexcitation of the atoms between $|1\rangle$ and $|r\rangle$ ) are correctly implemented, then the fidelity of our scheme is almost perfect ( $F=0.9998$ ) for $V_{\mathrm{DD}} T=2 \pi \times 4$ with the same parameters as in Fig. 5. To examine the advantages of our scheme compared to the approach via direct coherent coupling at the Förster resonance [24], we then check the robustness 

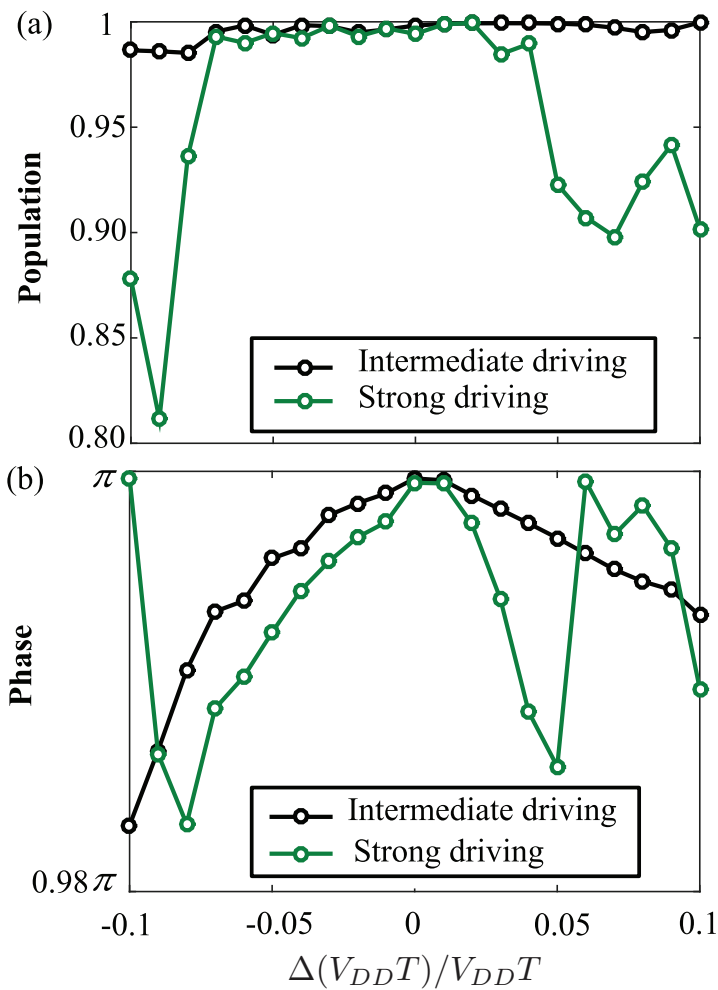

FIG. 6. (a) Population of the state $|g\rangle$ for the system initially in the state $|g\rangle$ against the dimensionless rescaled time deviation from the preset interaction time in the intermediate (black line) and strong (green line) driving regimes. (b) Phase of $|g\rangle$ against the dimensionless rescaled time deviation. Parameters are the same as those in Figs. 4(g)-4(i) (for strong driving) and Figs. 5(g)-5(i) (for intermediate driving).

of the two schemes to the fluctuation of the rescaled time for gate operation, as shown in Fig. 7. It is clearly verified that the fidelity of our scheme is less sensitive to the uncertainty of the operation time in contrast to the coherent coupling method with a similar gate duration. A fidelity as high as 0.995 can be well maintained for a time deviation of $\Delta\left(V_{\mathrm{DD}} T\right) /\left(V_{\mathrm{DD}} T\right) \sim$ $10 \%$ via the periodic Landau-Zener control, while the result

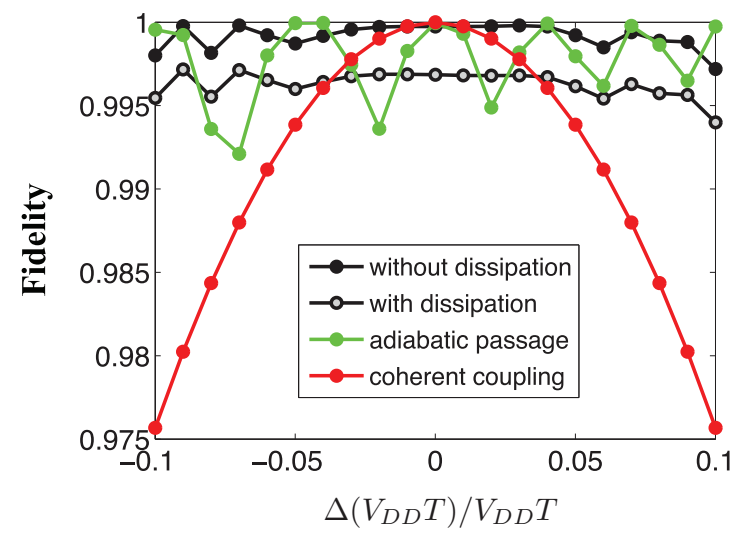

FIG. 7. Fidelities of the $\mathrm{CZ}$ gates vs the dimensionless rescaled time deviation for the schemes through the LZS approach with and without atomic spontaneous emission, the direct coherent coupling, and the adiabatic passage in Ref. [28]. See the main text for detail. obtained by the coherent coupling method is about 0.975 , which may be affected by additional errors for tuning the system exactly to the Förster resonance. Furthermore, the nonlinear driving scheme requires precise control of the two symmetric adiabatic sequences according to the power-law function $\delta_{1,2}(t)=s_{1}\left(t-t_{1,2}\right)+s_{2}\left(t-t_{1,2}\right)^{5}$, where the optimized set of parameters are $s_{1} / 2 \pi=-10 \mathrm{MHz} / \mu \mathrm{s}, s_{2} / 2 \pi=$ $-2600 \mathrm{MHz} / \mu \mathrm{s}^{5}, T=1.8 \mu \mathrm{s}, t_{1}=T / 4, t_{2}=3 T / 4$, and $V_{\mathrm{DD}} / 2 \pi=2.1 \mathrm{MHz}$ (see Ref. [28]). When the operation time for each of the two sequences deviates from the expected value by $\Delta T / T \sim 2 \%$, the fidelity will reduce to $\sim 0.995$. In contrast, the LZS-based scheme is more robust to imperfect timing.

In the context of Rydberg experiments, we simply take the example of the pair-state interaction channel $\left|90 S_{1 / 2}\right\rangle+$ $\left|96 S_{1 / 2}\right\rangle \rightarrow\left|90 P_{1 / 2}\right\rangle+\left|95 P_{1 / 2}\right\rangle$ in Cs Rydberg atoms, as previously found by Beterov et al. [28]. In this case, the atoms can be addressed individually since they are excited to different Rydberg states. The Förster interaction between the pair states has the energy defect $\delta_{0} / 2 \pi=75.6 \mathrm{MHz}$ and the exact Förster resonance occurs with the electric field being tuned to $E=29.75 \mathrm{mV} / \mathrm{cm}$. On the other hand, to meet the requirement of the intermediate-driving regime (see Fig. 5), the dipole-dipole matrix elements for the two atoms along the $z$ axis should be $V_{\mathrm{DD}} / 2 \pi \propto C_{3} / R^{3} \sim 3.2 \mathrm{MHz}$, which for this channel is equivalent to the interatomic distance $R=$ $20 \mu \mathrm{m}$ for $C_{3}=-154968 \mathrm{MHz} / \mu \mathrm{m}^{3}$. Correspondingly, the frequency of the $\mathrm{rf}$ driving field is $\omega / 2 \pi \sim 2.4 \mathrm{MHz}$, which is easy to access in experiments. Note that other transition channels related to this Förster resonance are safely neglected because of the large energy defects, which are the order of several hundred $\mathrm{MHz}$ [40].

The Rydberg states we considered have lifetimes around [40] $\tau_{90 S}=270 \mu \mathrm{s}, \tau_{96 S}=314 \mu \mathrm{s}, \tau_{90 P}=361 \mu \mathrm{s}$, and $\tau_{95 P}=406 \mu \mathrm{s}$ at room temperature $(\sim 300 \mathrm{~K})$. Thus, the two atoms excited to Rydberg states are subjected to atomic spontaneous emission during the LZS control. The effect of dissipation during the gate operation can be evaluated by using the conditional Hamiltonian

$$
\hat{H}_{\mathrm{cond}}=\hat{H}(t)-\frac{i}{2} \sum_{r} \gamma_{r}\left(\hat{\sigma}_{r r}^{(1)}+\hat{\sigma}_{r r}^{(2)}\right),
$$

where $\hat{\sigma}_{r r}^{(j)}=|r\rangle_{j j}\langle r|(j=1,2)$ and the sum is taken over all Rydberg states of the Förster interaction channel. The numerical estimate with the conditional Hamiltonian simply discards the state components with each of these two atoms going back to the computational space due to the spontaneous emission, which may have some overlap with the desired output state. Therefore, it provides a conservative result on the gate fidelity. As shown in Fig. 7, we can see that the spontaneous decay slightly reduces the gate fidelity, which, however, still surpasses 0.995 in general within $10 \%$ of the deviation of the rescaled time.

In terms of the typical parameters with respect to Cs Rydberg atoms, we now discuss the sensitivity of our scheme to fluctuations of the driving parameters. As shown in Fig. 8(a), there are ridges of high fidelity, which have the separation in detuning exactly given by the driving frequency $\omega$ and correspond to the multi-rf-photon resonance condition 

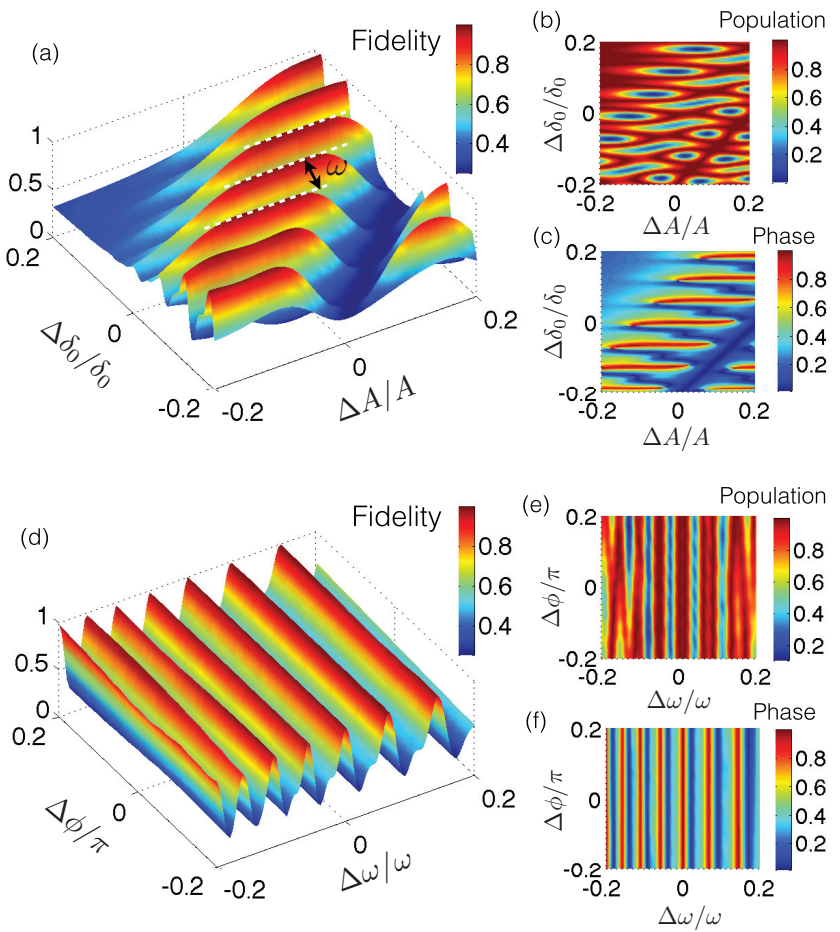

FIG. 8. Robustness of the fidelity of the $\mathrm{CZ}$ gate, population revival, and phase accumulation (divided by $\pi$ ) with respect to deviations of the driving amplitude $\Delta A / A$ and the detuning $\Delta \delta_{0} / \delta_{0}$ [(a)-(c)], and deviations of the driving frequency $\Delta \omega / \omega$ and the initial driving phase $\Delta \phi / \pi[(\mathrm{d})-(\mathrm{f})]$. Parameters are the $C_{3}$ coefficient of the dipole-dipole matrix elements $-154968 \mathrm{MHz} / \mu \mathrm{m}^{3}$, the interatomic distance $R=20 \mu \mathrm{m},\left(A, \delta_{0}, \omega\right) / 2 \pi=(83.2,76.8,3.15)$ $\mathrm{MHz}$, and the spontaneous decay rates $\left(\gamma_{90 S}, \gamma_{96 S}, \gamma_{90 P}, \gamma_{95 P}\right)=$ $(1 / 270,1 / 314,1 / 361,1 / 406) \mathrm{MHz}$.

$\delta_{0}=m \omega$. Therefore, the scheme requires accurate control of the driving frequency (typically limited by $\Delta \omega \sim \Omega_{\text {eff }} /|m|$ [37]) although the energy defect $\delta_{0} \pm \Delta \delta_{0}$ allows the two collective states to transit in between via different resonant channels [see Fig. 8(d)]. However, both the population and phase of the state show robustness against small fluctuations in the driving amplitude [see Figs. 8(b) and 8(c)], e.g., a deviation of the amplitude $\triangle A / A \approx 5 \%$ leads to the reduced fidelity $F \approx 0.992$, which is comparable to that of the nonlinear driving scheme with $5 \%$ deviations in $s_{1,2}$. In addition, the population and phase of the state are highly robust to the initial phase deviation of the rf field [see Figs. 8(e) and 8(f)].

Furthermore, our theoretical model can be related to the previously experimental demonstrations of the radiofrequency-assisted Förster resonances $n P_{3 / 2}+n P_{3 / 2} \rightarrow$ $n S_{1 / 2}+(n+1) S_{1 / 2}$ for $n<39$ in $\mathrm{Rb}$ atoms [25,36], where the periodic-driving-induced single- and multi-rf-photon transition can be alternatively explained in terms of the Floquet sidebands [33,38]. Considering the case that the nonzero Förster defect $\delta_{0}$ is now Stark-tuned by the composite electric field consisting of $\mathrm{dc}$ and $\mathrm{rf}$, $E=E_{\mathrm{dc}}+E_{\mathrm{rf}} \cos (\omega t)$. Then, the time-varying detuning between the pair of collective Rydberg levels is approximately given by $\delta(t) \approx \delta_{0}^{\prime}+A^{\prime} \cos (\omega t)$ for weak rf fields $E_{\mathrm{rf}} \ll E_{\mathrm{dc}}$, where $\delta_{0}^{\prime}=\delta_{0}+\left(\alpha_{n P}-\frac{1}{2} \alpha_{n s}-\frac{1}{2} \alpha_{(n+1) s}\right) E_{\mathrm{dc}}^{2}$, $A^{\prime}=2 E_{\mathrm{rf}} E_{\mathrm{dc}}\left(\alpha_{n P}-\frac{1}{2} \alpha_{n s}-\frac{1}{2} \alpha_{(n+1) s}\right)$, and $\alpha_{n l}$ are the quadratic polarizabilities. By assuming $\delta_{0}^{\prime}=m \omega$ with $m$ being a nonzero integer, we find that the robust $\mathrm{CZ}$ gate can be implemented in the intermediate-driving regime (i.e., $A^{\prime} / \delta_{0}^{\prime} \sim 1$ ) if and only if $\delta_{0} / m \omega \gg 3 / 2$. For $n=37$ [25,36], the parameter regime in Figs. 5(g)-5(i) corresponds to $\omega / 2 \pi=1 \mathrm{MHz}, E_{\mathrm{dc}} \approx 1.69 \mathrm{~V} / \mathrm{cm}$ with $E_{\mathrm{rf}} / E_{\mathrm{dc}}=0.1$.

\section{v. CONCLUSION}

In summary, we have proposed a feasible experimental scheme for implementation of a two-qubit logic gate by modulating the Förster resonance with a periodic-driving field. The Stark-tuned Förster interaction between the two pairs of Rydberg states can be regarded as a periodically driven two-level system, with a time-invariant coupling strength and a sinusoidal time-dependent detuning. The results show that the gate can be accomplished within an operation time comparable to that required by the method based on double adiabatic passages [28], and in contrast to the coherent coupling scheme, the gate fidelity is much less sensitive to the fluctuations of the interaction time and the motion-sensitive dipole-dipole matrix elements due to the sequential Landau-Zener transitions. We numerically analyze the implementation of this gate with the realistic Förster interaction channel in Cs Rydberg atoms, and the results demonstrate its performance is insensitive to both the time fluctuations and atomic spontaneous emission, confirming its promise in quantum information processing.

\section{ACKNOWLEDGMENTS}

W.L. acknowledges support from the UKIERI-UGC Thematic Partnership No. IND/CONT/G/16-17/73, and EPSRC Grant No. EP/M014266/1 and EP/R04340X/1. L.-T.S., H.W., and S.-B.Z are supported by the National Natural Science Foundation of China under Grants No. 11774058, No. 11674060, No. 11874114, and No. 11705030, the Natural Science Foundation of Fujian Province under Grant No. 2017J01401, and the Qishan fellowship of Fuzhou University. H.W. acknowledges particularly the financial support by the China Scholarship Council for the academic visit to the University of Nottingham.
[1] M. Saffman, T. G. Walker, and K. Mølmer, Rev. Mod. Phys. 82, 2313 (2010).

[2] A. Browaeys, D. Barredo, and T. Lahaye, J. Phys. B 49, 152001 (2016).
[3] D. Jaksch, J. I. Cirac, P. Zoller, S. L. Rolston, R. Cote, and M. D. Lukin, Phys. Rev. Lett. 85, 2208 (2000).

[4] M. Müller, I. Lesanovsky, H. Weimer, H. P. Büchler, and P. Zoller, Phys. Rev. Lett. 102, 170502 (2009). 
[5] D. D. Bhaktavatsala Rao and K. Mølmer, Phys. Rev. A 89, 030301(R) (2014).

[6] T. Keating, R. L. Cook, A. M. Hankin, Y.-Y. Jau, G. W. Biedermann, and I. H. Deutsch, Phys. Rev. A 91, 012337 (2015).

[7] L. Isenhower, E. Urban, X. L. Zhang, A. T. Gill, T. Henage, T. A. Johnson, T. G. Walker, and M. Saffman, Phys. Rev. Lett. 104, 010503 (2010).

[8] X. L. Zhang, A. T. Gill, L. Isenhower, T. G. Walker, and M. Saffman, Phys. Rev. A 85, 042310 (2012).

[9] M. M. Müller, M. Murphy, S. Montangero, T. Calarco, P. Grangier, and A. Browaeys, Phys. Rev. A 89, 032334 (2014).

[10] K. M. Maller, M. T. Lichtman, T. Xia, Y. Sun, M. J. Piotrowicz, A. W. Carr, L. Isenhower, and M. Saffman, Phys. Rev. A 92, 022336 (2015).

[11] S.-L. Su, Y. Gao, E. Liang, and S. Zhang, Phys. Rev. A 95, 022319 (2017).

[12] H. Wu, X.-R. Huang, C.-S. Hu, Z.-B. Yang, and S.-B. Zheng, Phys. Rev. A 96, 022321 (2017).

[13] L. S. Theis, F. Motzoi, F. K. Wilhelm, and M. Saffman, Phys. Rev. A 94, 032306 (2016).

[14] M. H. Goerz, E. J. Halperin, J. M. Aytac, C. P. Koch, and K. B. Whaley, Phys. Rev. A 90, 032329 (2014).

[15] D. Petrosyan, F. Motzoi, M. Saffman, and K. Mølmer, Phys. Rev. A 96, 042306 (2017).

[16] I. I. Beterov, M. Saffman, E. A. Yakshina, V. P. Zhukov, D. B. Tretyakov, V. M. Entin, I. I. Ryabtsev, C. W. Mansell, C. Mac Cormick, S. Bergamini and M. P. Fedoruk, Phys. Rev. A 88, 010303(R) (2013).

[17] X.-Q. Shao, D.-X. Li, Y.-Q. Ji, J.-H. Wu, and X.-X. Yi, Phys. Rev. A 96, 012328 (2017).

[18] D. Møller, L. B. Madsen, and K. Mølmer, Phys. Rev. Lett. 100, 170504 (2008).

[19] S.-L. Su, E. Liang, S. Zhang, J.-J. Wen, L.-L. Sun, Z. Jin, and A.-D. Zhu, Phys. Rev. A 93, 012306 (2016).

[20] Y. H. Kang, Y. H. Chen, Z. C. Shi, B. H. Huang, J. Song, and Y. Xia, Phys. Rev. A 97, 042336 (2018).

[21] T. Förster, Ann. Phys. (NY) 437, 55 (1948).

[22] I. I. Ryabtsev, D. B. Tretyakov, I. I. Beterov, and V. M. Entin, Phys. Rev. Lett. 104, 073003 (2010).
[23] H. Gorniaczyk, C. Tresp, P. Bienias, A. Paris-Mandoki, W. Li, I. Mirgorodskiy, H. P. Büchler, I. Lesanovsky, and S. Hofferberth, Nat. Commun. 7, 12480 (2016).

[24] S. Ravets, H. Labuhn, D. Barredo, L. Béguin, T. Lahaye, and A. Browaeys, Nat. Phys. 10, 914 (2014).

[25] E. A. Yakshina, D. B. Tretyakov, I. I. Beterov, V. M. Entin, C. Andreeva, A. Cinins, A. Markovski, Z. Iftikhar, A. Ekers, and I. I. Ryabtsev, Phys. Rev. A 94, 043417 (2016).

[26] M. D. Lukin, M. Fleischhauer, R. Cote, L. M. Duan, D. Jaksch, J. I. Cirac, and P. Zoller, Phys. Rev. Lett. 87, 037901 (2001).

[27] H.-Z. Wu, Z.-B. Yang, and S.-B. Zheng, Phys. Rev. A 82, 034307 (2010).

[28] I. I. Beterov, M. Saffman, E. A. Yakshina, D. B. Tretyakov, V. M. Entin, S. Bergamini, E. A. Kuznetsova, and I. I. Ryabtsev, Phys. Rev. A 94, 062307 (2016).

[29] I. I. Beterov, G. N. Hamzina, E. A. Yakshina, D. B. Tretyakov, V. M. Entin, and I. I. Ryabtsev, Phys. Rev. A 97, 032701 (2018).

[30] A. Fioretti, D. Comparat, C. Drag, T. F. Gallagher, and P. Pillet, Phys. Rev. Lett. 82, 1839 (1999).

[31] N. Saquet, A. Cournol, J. Beugnon, J. Robert, P. Pillet, and N. Vanhaecke, Phys. Rev. Lett. 104, 133003 (2010).

[32] S.-S. Zhang, W. Gao, H. Cheng, L. You, and H. P. Liu, Phys. Rev. Lett. 120, 063203 (2018).

[33] C. S. E. van Ditzhuijzen, A. Tauschinsky, and H. B. van Linden van den Heuvell, Phys. Rev. A 80, 063407 (2009).

[34] D. M. Berns, W. D. Oliver, S. O. Valenzuela, A. V. Shytov, K. K. Berggren, L. S. Levitov, and T. P. Orlando, Phys. Rev. Lett. 97, 150502 (2006).

[35] S. Basak, Y. Chougale, and R. Nath, Phys. Rev. Lett. 120, 123204 (2018).

[36] D. B. Tretyakov, V. M. Entin, E. A. Yakshina, I. I. Beterov, C. Andreeva, and I. I. Ryabtsev, Phys. Rev. A 90, 041403(R) (2014).

[37] S. Ashhab, J. R. Johansson, A. M. Zagoskin and F. Nori, Phys. Rev. A 75, 063414 (2007).

[38] S. N. Shevchenko, S. Ashhab, and F. Nori, Phys. Rep. 492, 1 (2010).

[39] G. Heinrich, J. G. E. Harris, and F. Marquardt, Phys. Rev. A 81, 011801(R) (2010).

[40] I. I. Beterov, I. I. Ryabtsev, D. B. Tretyakov, and V. M. Entin, Phys. Rev. A 79, 052504 (2009). 